D

KU Leuven, Department of Rehabilitation Sciences, Leuven, Belgium and Respiratory and Pulmonary Rehabilitation Division, University Hospitals Gasthuisberg, Leuven, Belgium

\title{
Should European Respiratory Society meetings come with a health warning?
}

It is now widely recognised that a lack of physical activity increases the risk of chronic diseases including cardiovascular disease, diabetes, osteoporosis and obesity [1]. Several of these diseases are prevalent comorbidities in patients with chronic respiratory disease, known to be inactive. Enhancing physical activity is therefore one of the five priorities in the prevention of morbidity of non-communicable diseases [2]. Over previous years, it has been shown that increasing physical activity in patients with respiratory diseases is challenging. Specific interventions geared to change patient behaviour show promise. Step counters are often used in these interventions and telecoaching platforms, including smartphones to provide feedback and feedforward in patients may offer a platform to integrate activity enhancing interventions successfully.

It was in the context of one such study (NCT02158065, part of the www.PROactivecopd.com study portfolio) that I personally got engaged with step counters. This study used a complex intervention including a smartphone and a step counter (Fitbug Air; Fitbug, London, UK) to enhance physical activity of patients with COPD. Some technical difficulties occurred in the first few patients. So, in order to have first-hand information about the functionality of the technology platform, I volunteered to wear the step counter and use the smartphone for the duration of the study and, thereafter, for 1 year of data collection. Insight into my day-to-day physical activity profile provided an interesting personal perspective on physical activity, a topic I have been studying for several years.

Of a total of 365 days, the step counter did not provide data on only 3 days (reasons were forgetting to clip the step counter and battery issues). My number of steps was $8523 \pm 2637$ steps $\cdot$ day $^{-1}$ (median (interquartile range) 8106 (659410141.00) steps.day ${ }^{-1}$ ). In general, and contrary to observations in patients with chronic obstructive pulmonary disease, weekends (median 9135 steps day $^{-1}$ ) proved more active than week-

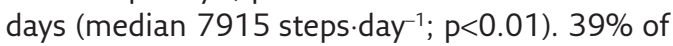
weekend days had $>10000$ steps (active days), whereas only $23 \%$ of weekdays were "active". $43 \%$ of weekdays had step counts $<7500$ steps, used as a benchmark for too low physical activity levels [3].

The number of steps was highly variable from day to day (coefficient of variation was 0.31). During holidays, step counts were higher than usual (median steps 9137 (6971-11772)) (figure 1). Interestingly, as a member of the Executive Committee of the European Respiratory Society (ERS), I analysed my physical activity on days with scheduled ERS activities ( $n=16$ days, including the ERS conference, Executive Committee meetings in Geneva, and Zurich, HERMES (Harmonised Education in Respiratory Medicine for European Specialists) meeting in Leuven and the ERS Annual Presidential Summit in Brussels); sadly, step counts were significantly less on these days and, on the average, fell in the range of "low physical activity" (6557 (5090-7904) steps.day ${ }^{-1}$ )
Cite as: Troosters T. Should European Respiratory Society meetings come with a health warning? Breathe 2015: 11: 279-281.

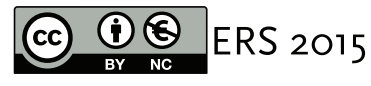

HERMES syllabus link: modules E.1.14, E.1.21 
(figure 1). These step counts would be compatible with a Global Initiative for Chronic Obstructive Lung Disease (GOLD) stage II patient and scientists in the field of physical activity would call to "urgent action" to improve physical activity [4].

These observations might constitute a call for action for organisations that organise day-long meetings with lack of physical activity. Engaging in physical activity is obviously a personal choice and a personal responsibility; however, the introduction of group physical activities like a group walk would constitute a healthy and useful break of an otherwise inactive day. A 30-min walk would increase step counts by approximately 3000 steps.day ${ }^{-1}$. As such, the organisation could increase the awareness around physical activity for their leadership. Such an initiative was taken by the American Heart Association, for whom 1850 delegates wore a pedometer and steps were recorded during their Scientific Council. Together, they collected 10.7 million steps over 2.5 days [5]. With physical activity as a general theme for the 2015 Amsterdam congress, the ERS leadership and ERS members would benefit from taking the active option, every single day, and particularly during ERS events. After banning smoking from ERS International Congresses, a next step could be to develop a supportive exercise and physical activity culture for conference attendees. Importantly, it has been shown that physically active clinicians have more confidence to provide exercise counselling to their patients. Hence, being an active healthcare professional is a good first step to being a better clinician [6]. As such, a goal of Congress would be met: providing better care! Admittedly I, myself, have work to do!

Wearing a step counter was an excellent first step to make myself aware of my own lack of physical activity. Consumer devices nowadays are relatively inexpensive, have sufficient reliability and can help to provide reliable feedback on physical activity
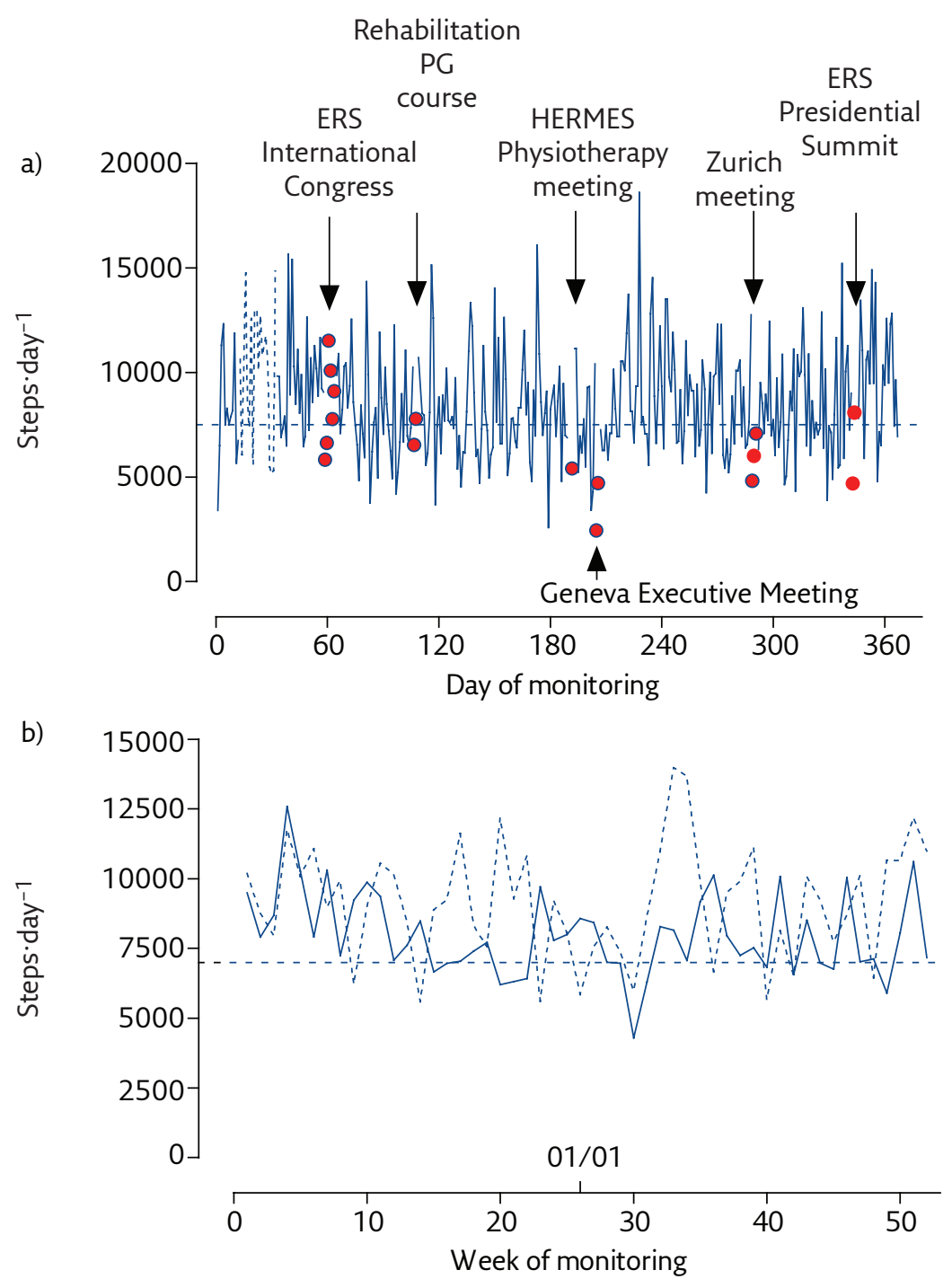

Figure 1 Daily (upper) and weekly (Lower panel) averages of my physical activity profile. Upper Panel includes days with ERS activities as red dots. Lower panel provides information on week (solid line) and weekend (dashed lines). The First of January (01/01) is indicated for reference. 
[7]. Although the 10000 steps day $^{-1}$ goal may not be the best from an academic perspective as it ignores the fact that activities should be carried out at moderate intensity, it provides an easy-to-use surrogate measure of having an active life. A step count $<7500$ steps day $^{-1}$ is suggested to be too low to maintain good health in the long term [3]. The World Health Organization advocates to do $>150$ mins of moderate-intensity aerobic physical activity ( $>3$ metabolic equivalents; walking 4-6 $\mathrm{km} \cdot \mathrm{h}^{-1}$ ) throughout the week or to do $>75$ mins of vigorous-intensity aerobic physical activity throughout the week or an equivalent combination of moderate- and vigorousintensity activity [8].

In summary, an affordable simple step counter provided useful insight in my personal physical activity profile and identified weekends (with a 3.5 year old child at home) to be more active, whereas participation in day-long meetings was detrimental for my physical activity profile. Such meetings should not come with a health warning, but rather societies organising such meetings should make them as healthy as possible by trying to embed attractive and client centered physical activity options during their meeting breaks to facilitate and promote physical activity among their meeting participants.

\section{Acknowledgements}

Ben Nemery is acknowledged for his suggestions to enhance the quality of this article. Funded by Flemish Research Agency G.0871.13.

\section{Conflict of interest}

None declared.

\section{References}

1. Lee IM, Shiroma EJ, Lobelo F, et al. Effect of physical inactivity on major non-communicable diseases worldwide: an analysis of burden of disease and life expectancy. Lancet 2012; 380: 219-229

2. Beaglehole R, Bonita R, Horton R, et al. Priority actions for the non-communicable disease crisis. Lancet 2011; 377: 1438-1447.

3. Tudor-Locke C, Craig CL, Brown WJ, et al. How many steps/ day are enough? For adults. Int J Behav Nutr Phys Act 2011; 8: 1521-1537.

4. Watz H, Pitta F, Rochester CL, et al. An official European Respiratory Society statement on physical activity in COPD. Eur Respir J 2014; 44: 79.
5. Harrington RA, Arena R, Despres JP, et al. More than 10 million steps in the right direction: results from the first American Heart Association scientific sessions walking challenge. Prog Cardiovasc Dis 2015; 57: 296-298.

6. Howe M, Leidel A, Krishnan SM, et al. Patient-related diet and exercise counseling: do providers' own lifestyle habits matter? Prev Cardiol 2010; 13: 180-185.

7. Case MA, Burwick HA, Volpp KG, et al. Accuracy of smartphone applications and wearable devices for tracking physical activity data. JAMA 2015; 313: 625-626.

8. World Health Organization. Global recommendations on Physical Activity for health. WHO, Geneva, 2010. 